\title{
Double staining protocol for developing European sea bass (Dicentrarchus labrax) larvae
}

\author{
By M. J. Darias ${ }^{1, *}$, O. Lan Chow Wing ${ }^{1}$, C. Cahu ${ }^{1}$, J. L. Zambonino-Infante ${ }^{1}$ and D. Mazurais ${ }^{1}$
}

\author{
${ }^{1}$ Ifremer Marine Fish Nutrition Team, Nutrition Aquaculture and Genomics Research Unit, UMR 1067. \\ Ifremer, Technopole Brest-Iroise, BP 70, 29280 Plouzané, France
}

\author{
*: Corresponding author present address : M. J. Darias, IRTA-SCR, Ctra. de Poble Nou s/n, km 5.5, 43450, Sant \\ Carles de la Ràpita, Tarragona, Spain, email address : maria.darias@irta.cat
}

\begin{abstract}
:
The alcian blue-alizarin red technique was successfully adjusted to stain developing European sea bass (Dicentrarchus labrax) larvae. For an optimal staining protocol design both larval size and their morphological characteristics at each developmental stage were considered, since such parameters notably influence the staining of tissues. The incubation times of the different solutions were adjusted to allow the stain penetration for revealing the integrity of cartilaginous and bony tissues without significant tissue degradation. Three developmental windows were determined for an optimal staining procedure: (i) $4.5-6.4 \mathrm{~mm}$, (ii) $6.7-8.7 \mathrm{~mm}$, and (iii) $12.8-15.5 \mathrm{~mm}$ total length (TL). In order to validate the continuity of staining along the larval development, quantification of bone mineralization and osteocalcin gene expression were also monitored. Quantitative analysis revealed that ossification followed an exponential kinetic that was positively correlated with the osteocalcin gene expression pattern (Rs $=0.9762, \mathrm{P}<0.05$ ). The mineralized tissue increased from $6.4 \mathrm{~mm}$ TL onwards, corresponding with the detection of the first ossified structures. The quantity of bony tissue increased gradually until $7.6 \mathrm{~mm}$ TL, since mineralization remained limited to the skull. From 8.3 to $15.5 \mathrm{~mm} \mathrm{TL}$, the mineralized bone was notable and nearly concerned the whole larval skeleton (skull, vertebral column and caudal complex). Since it was possible to detect the first cartilaginous and mineralized structures in specimens as small as 4.5 and $6.4 \mathrm{~mm} \mathrm{TL}$, respectively, this procedure is a useful tool to study the European sea bass skeletal ontogenesis, to precociously diagnose skeletal malformations in small larvae and eventually to better characterize the effect of different environmental and/or nutritional factors on the ossification status of specific skeletal components.
\end{abstract}


42 The use of alcian blue-alizarin red double staining methodology to stain fish is

43 relatively old (Dingerkus and Uhler, 1977; Potthoff, 1984; Taylor and Van Dyke,

44 1985) and it has been used to study the skeletal development in several marine fish

45 species of the Mediterranean aquaculture such as Sparus aurata (Faustino and

46 Power, 1998, 1999, 2001), Dentex dentex (Koumoundouros et al., 2000),

47 Scophthalmus maximus (Wagemans et al., 1998) or Solea senegalensis (Gavaia et

48 al., 2002). Moreover, this technique allowed detecting and characterizing skeletal

49 abnormalities in reared fish species (Daoulas et al., 1991; Marino et al., 1993;

50 Koumoundouros et al., 1997a,b, 2002; Gavaia et al., 2002; Fernández et al., 2008,

51 2009; Mazurais et al., 2008, 2009; Darias et al., 2010), which cause severe

52 economic impact for the aquaculture industry. There are different causative factors,

53 including physiological, environmental, genetic, xenobiotic and nutritional ones,

54 affecting the larval and juvenile stages of cultured freshwater and marine fish (Lall

55 and Lewis-McCrea, 2007). Recently, this double staining procedure has also been

56 used as a tool to evaluate the nutritional effects on the quality of the fish skeleton at

57 the end of the larval period (Fernández et al., 2008, 2009; Mazurais et al., 2008,

58 2009; Darias et al., 2010). However, since nutritional needs change through the

59 larval development, the precocious detection of skeletal deformities could aid to

60 determine the influence nutrients on early larval development. In this sense, the

61 establishment of the alcian blue-alizarin red double staining technique for

62 developing European sea bass larvae becomes useful to describe skeletogenesis

63 as well as to evaluate any factor that could induce skeletal deformities. Although the

64 ontogeny of the cephalic (Gluckmann et al., 1999) and appendicular (Marino et al.,

65 1993) skeleton has been investigated in this species, there is no information about

66 the characterization of the ossification process using a quantitative methodology.

67 Quantification of bone mineralization could also serve to determine and localize 
68 possible disruptions during this process that could constitute the origin of skeletal

69 deformities. In order to validate bone quantification analysis based on the double

70 staining approach, it was found appropriated to study in parallel the expression

71 pattern of the osteocalcin gene, which serves as marker for the mineralization

72 process. Osteocalcin (Bone Gla protein) is indeed the most abundant non

73 collagenous protein in the extracellular matrix of bony tissues (Nishimoto et al.,

74 1992), it is synthesized by matures osteoblasts and constitutes nowadays a marker

75 for bone remodelling in various vertebrates (Swaminathan, 2001; Nishimoto et al.

76 2003, Benhamou 2007).

\section{Material and methods}

80 Rearing conditions and larval sampling

82 European sea bass larvae were obtained from the Ecloserie Marine de Gravelines

83 (Gravelines, France). Larvae were acclimated and divided into four 35-liter

84 cylindroconical fiberglass tanks (2,100 larvae per tank) at an initial density of 60

85 larvae per litre. Throughout the experiment, temperature was $20^{\circ} \mathrm{C}$, salinity was

$8635 \%$, and the oxygen level was maintained above $6 \mathrm{mg}$ per litre. Photoperiod was

$8724: 0$ hours light-dark cycle, and maximum light intensity was 9 watts per square

88 meter at the water surface. Larvae were fed from day 6 to day 45 post hatching

89 (dph) on microparticulate diets (WO 0064273) prepared in our laboratory as

90 described by Cahu et al. (2003). Forty to fifty larvae were sampled from each tank at

$917,11,15,17,21,25,30,35$ and $40 \mathrm{dph}$ for double staining, which corresponded to

$924.5,5.4,6.4,6.7,7.6,8.3,12.8,14$ and $15.5 \mathrm{~mm} \mathrm{TL}$, respectively.

93

$94 \quad$ Alcian blue-Alizarin red double staining

95 
96 The alcian blue-alizarin red double staining technique was adjusted to stain

97 cartilaginous and bony tissue structures in developing European sea bass larvae as

98 next described.

99

100 Fixation: forty to fifty larvae were sampled from each tank and preserved in fixative

101 solution ( $4 \%$ formalin buffered to $\mathrm{pH} 7$ with $0.1 \mathrm{M}$ phosphate buffer) for at least 24

102 hours.

103 Washing: all larval groups were transferred to hand-made sieves and placed into a

104 big glass of Pyrex to facilitate the change of solutions and to treat them at the same

105 time. Larvae were incubated in distilled water until they sank. Afterwards, larvae

106 were washed in distilled water two times 5 minutes each.

107 Cartilage staining: larvae were transferred into an alcian blue (Alcian blue 8GX,

108 SIGMA A5268) solution (100 mg/l alcian blue, $800 \mathrm{ml} / \mathrm{l} 95 \%$ ethanol, and $200 \mathrm{ml} / \mathrm{l}$

109 acetic acid) and the incubation time varied according to the larval size until the

110 achievement of the staining saturation (Table 1).

111 Neutralization: the remaining acid of larval tissues was neutralized by incubating

112 specimens during 3 minutes in a solution containing $100 \%$ ethanol in $1 \% \mathrm{KOH}$.

113 Rehydration: larvae were rehydrated in decreasing ethanol series $(95,70,40,15$

$114 \%)$, two times 15 minutes each, and in distilled water until larvae sank. Finally,

115 larvae were incubated in distilled water two times 5 minutes each.

116 Bleaching: pigmented larvae were incubated in a bleaching solution (1 volume 3\%

$117 \mathrm{H}_{2} \mathrm{O}_{2}$ and 9 volumes $1 \% \mathrm{KOH}$ ) during a variable time, according to the degree of 118 pigmentation and size (Table1).

119 Clearing: ossified larvae were incubated in a rinsing solution (7 volumes distilled

120 water, 3 volumes sodium borate and 0.5-2.5 g trypsin -SIGMA T-4799-) for 20 121 hours. 
122 Bone staining: larvae were incubated in alizarin red (SIGMA T4799) solution (5 g/l

123 alizarin red in $1 \% \mathrm{KOH}$ ) during various periods of time, depending on the ossification

124 degree (Table 1).

125 Washing: larvae were washed with distilled water and subsequently with a solution

126 of $1 \% \mathrm{KOH}$ until the elimination of staining background. The incubation time varied

127 according to the degree of ossification (Table 1).

128 Dehydration: larvae were incubated in the following increasing series of glycerol +

$1291 \% \mathrm{KOH}: 2$ hours in 40\% Glycerol $+60 \% 1 \% \mathrm{KOH}$ and 6 hours in $70 \%$ Glycerol + $13030 \% 1 \% \mathrm{KOH}$.

131 Stocking: stained larvae were preserved in $100 \%$ glycerol.

133 Image analysis

135 Stained larvae were placed on Petri dishes containing glycerol and scanned (Epson

136 Perfection 4990 Photo; Light source: white cold cathode fluorescent lamp) to create

137 a 2,500-kb picture. The results were compiled and statistically analyzed as

138 described below. Individual size and the surfaces corresponding to cartilage and

139 bone in whole larvae were visualized and quantified using a computerized image

140 analysis package (IMAQ Vision Builder, National Instruments, Austin, TX). The

141 scripting feature of IMAQ Vision Builder was used to record a series of image-

142 processing steps and their specific parameters, so that the computerized image

143 analyses were also performed simultaneously for all samples (batch processing).

144 The script used a list of image-processing commands encompassing the selection of

145 pixel color range and quantification. Selecting ranges of pixel values in color images

146 (threshold operations) allowed the pixels associated with red (bone) or blue

147 (cartilage) staining to be distinguished. The number of selected pixels was then 148 quantified using a particle analyses operation. The value of red pixels was 149 associated to the degree of bone mineralization. 
151 Gene expression

153 Total RNA from whole larvae was extracted using TRIzol (Invitrogen) and reverse-

154 transcribed (iScript cDNA Synthesis Kit, Bio-Rad Laboratories) to measure the

155 expression of Osteocalcin (AY663813). Quantitative PCR analyses were performed 156 in triplicate using iQ SYBR Green supermix 2X (Bio-Rad Laboratories). Ef1 was

157 chosen as a housekeeping gene (AJ866727). Gene primer sequences, thermal 158 cycling, real-time PCR efficiencies and the relative quantity of target gene-specific 159 transcripts among samples were determined as described in Mazurais et al. (2008).

161 Statistics

162

Results are expressed as means \pm standard deviations. The correlation between osteocalcin expression and ossification degree was calculated using the Spearman's correlation index (Rs) with a significance level of $5 \%$.

164 Results and discussion

166 Alcian blue-alizarin red double staining protocol

168 The present double staining protocol for developing European sea bass larvae was 169 defined based on diverse published protocols (Dingerkus and Uhler, 1977; Park and 170 Kim, 1984; Potthoff, 1984; Taylor and Van Dyke, 1985; Gavaia et al., 2000). To 171 achieve optimal staining conditions, several incubation times of the different 172 solutions were tested according to larval size and developmental stage. Thus, a 173 compromise between colour saturation in cartilage and bone and the prevention of 174 tissue degradation was reached. The best staining results were obtained when 
175 larvae were divided in three developmental groups and treated as shown in Table 1.

176 This protocol allowed detecting cartilaginous and calcified skeletal structures from

$1774.5 \mathrm{~mm}$ and $6.4 \mathrm{~mm} \mathrm{TL}$, respectively (Fig. 1). In addition, it was also possible to

178 distinguish some deformities in the skull, vertebral column and caudal fin complex 179 (Fig. 2).

180 Double staining has been used to describe skeletogenesis and to detect skeletal 181 malformations in several fish species (Daoulas et al., 1991; Boglione et al., 2001; 182 Koumoundouros et al., 1997, 2002; Gavaia et al., 2002; 2006; Sfakianakis et al., 183 2004; Fernández et al., 2008; 2009; Mazurais et al., 2008; 2009; Darias et al., 184 2010). Gavaia et al. (2000) improved this technique to detect cartilage and bone in 185 Solea senegalensis, Sparus aurata, Diplodus sp. and Halobatrachus didactylus 186 larvae and juveniles as small as $2.6 \mathrm{~mm}$ notochord length (NL). Due to the 187 similarities shared in terms of larval size and species analysed, protocols of Potthoff 188 (1984) and Gavaia et al. (2000) were more closely examined than the others for the 189 adjustment of this double staining procedure in European sea bass, which 190 presented several methodological differences. For instance, specimens were 191 directly washed in distilled water rather than treat them with TBST (Tris-NaCl-Triton 192 X-100 solution) to eliminate the residual fixative. Potthof (1984) stated that a 193 dehydration step before cartilage staining is important since small amounts of water 194 interfere with the staining of cartilage. Nevertheless, the prevention of non-specific 195 stain observed by Gavaia et al. (2000) when larvae were kept hydrated prior the 196 alcian blue staining, rather than dehydrated or directly transferred from the fixative 197 solution, was considered in the present protocol, which gave satisfying results. The 198 incubation times in alcian blue solution of the different larval groups were similar to 199 those used for other fish species (Potthoff, 1984; Gavaia et al., 2000). Following the 200 recommendations of Gavaia et al. (2000), a $\mathrm{KOH}$ :ethanol solution was used to 201 neutralize the remaining alcian blue solution that could continue to demineralise the 202 larval tissues. The higher $\mathrm{pH}$ prevents further calcium loss from the bony tissues 
203 which is essential to obtain a suitable alizarin red stain. Larval tissues could also be

204 neutralized using a saturated sodium borate solution (Potthoff, 1984). However, the

205 main difference between the protocols was observed in the bleaching step. In this

206 study it was performed before bone staining, this being in agreement with Potthoff

207 (1984) and Taylor and Van Dyke (1985) and contrary to Dingerkus et al. (1977) and

208 Gavaia et al. (2000). The bleaching treatment was only used in older larvae since

209 they were more pigmented. This step was especially important for the subsequent

210 quantitative analysis of the ossification degree because the brown colour of the

211 pigmented skin interfered with the pixel color range selected to cover the ossified

212 bony tissue. It was necessary to increase the incubation time used for bone staining

213 to 20 hours in larvae longer than $12.8 \mathrm{~mm}$, coinciding with thicker tissues, to obtain

214 an adequate staining of ossified structures. This was in agreement with Potthoff

215 (1984) who found necessary $24 \mathrm{~h}$ to stain bony structures in fish larvae ranging from

21610 to $80 \mathrm{~mm}$ TL. However, Gavaia et al (2000) proposed 30 minutes for all treated

217 larvae ranging from 2.6 to $78 \mathrm{~mm}$. Such a notable difference in the incubation time

218 could be related with the absence of TBST treatment in the present protocol since,

219 as Gavaia et al. (2000) reported, it improves dye penetration. Finally, a treatment

220 with trypsin was necessary to clear larger European sea bass specimens, while this

221 was not required in other species of comparable size (Gavaia et al., 2000).

223 Bone mineralization and osteocalcin expression

225 To evaluate the ossification process, the total number of red pixels was counted

226 which represents the mineralization degree of bony tissue in each developmental

227 stage. The ossification degree of bony tissue increased from $6.4 \mathrm{~mm} \mathrm{TL}$ (15 dph)

228 onwards, coinciding with the detection of the first ossified structures (dentary,

229 maxillas and cleithrum). Bony tissue formed gradually until $7.6 \mathrm{~mm}$ TL (21 dph),

230 since mineralization remained limited to the skull. From $8.3 \mathrm{~mm}$ TL ( $25 \mathrm{dph}$ ) until 
$231 \quad 15.5 \mathrm{~mm}$ TL (40 dph), the mineralized bone was notable and nearly concerned the

232 whole larval skeleton (skull, vertebral column and caudal complex).

233 The spatio-temporal sequence of the bony structures formation was in accordance 234 with that obtained by Gluckmann et al. (1999). It was also verified that the 235 appearance of bony tissues was correlated with the increase of the ossification 236 degree measured in the different developmental stages. Quantitative analysis 237 indicated that ossification degree follows an exponential kinetic with an inflexion 238 point around $8.3 \mathrm{~mm} \mathrm{TL}$, this being associated with the sequence of ossification of 239 the skeletal elements. That is, before that size, mineralized structures mainly 240 corresponded to the skull while from $8.3 \mathrm{~mm}$ TL onwards, the centra of the vertebral 241 column extremely contributed to the observed ossification increase.

242 The different incubation times used at each developmental stage did not introduce 243 any bias in the pattern of larval staining degree. For instance, the use of trypsin only 244 in specimens from $12.8 \mathrm{~mm}$ TL onwards, or even the wide range of incubation times 245 of the alizarin red solution (30 minutes in larvae from 4.5 to $6.4 \mathrm{~mm}$ TL and 20 hours 246 in the other ones), did not influence the bone staining profile (Fig. 3).

247 European sea bass larvae showed an exponential pattern of osteocalcin expression 248 during larval development. This is in line with previous studies that have shown a 249 notable increase of osteocalcin expression from 22-25 dph onwards, coinciding with 250 mineralization of the vertebral column (Darias et al., 2010). Such profile was 251 positively correlated with that of the ossification degree determined by the double 252 staining approach (Fig. 3) $(\mathrm{Rs}=0.9762, \mathrm{P}<0.05)$. This result was expectable since 253 osteocalcin is implied in the differentiation and mineralization of osteoblasts (Lian 254 and Stein, 1995), the bone-forming cells (Fig. 3). Together with the strong similarity 255 existing between the kinetic of the ossification degree measured by the double 256 staining method and the osteocalcin expression pattern, these findings validate the 257 present protocol (Fig. 3). Mazurais et al. (2008) already observed a high correlation 258 between osteocalcin expression and red alizarin stain of mineralized bone tissue in 
38 day-old European sea bass larvae, demonstrating that this gene is a good

260 indicator of bone differentiation. The present study ratifies that osteocalcin

261 constitutes a suitable molecular marker for the ossification status in European sea

262 bass larvae, not only at the end of the larval period but throughout the larval

263 development.

265 In conclusion, the alcian-blue alizarin red technique was successfully adjusted for 266 developing European sea bass, allowing to detect cartilage and bone in larvae with

267 a minimum size of $4.5 \mathrm{~mm}$ and $6.4 \mathrm{~mm} \mathrm{TL}$, respectively, which denotes the 268 convenience of this method for skeletal development studies. Additionally, a 269 quantitative analysis of the ossification degree throughout the European sea bass

270 larval development based on this staining procedure was also achieved. This could 271 serve to determine and localize possible disruptions during the ossification process

272 that could constitute the origin of skeletal deformities. Finally, osteocalcin expression

273 has not only validated the bone quantification analysis based on the double staining

274 approach, but has also demonstrated to be a suitable molecular marker of the 275 presence of mineralized bone in developing European sea bass larvae. Therefore, 276 this is a useful tool to study the skeletal ontogenesis, to precociously diagnose 277 skeletal malformations in small specimens and eventually to better characterize the 278 effect of different environmental and/or nutritional factors on the ossification status of 279 specific skeletal components.

\section{Aknowledgements}

This work was, in part, supported by FINEFISH, a Collective Research Project of the sixth Framework Programme of the European Union (Contract 012451). M.J. Darias was supported by a postdoctoral fellowship from the Fundación Ramón Areces 
(Spain) and by a MICINN National Project (AGL2008-03897-C04-01) (Spain) to participate in the first IAFSB Workshop.

\section{References}

285 Benhamou, C.L., 2007. Effets des traitements de l'ostéoporose sur la qualité 286 osseuse. Revue du Rhumatisme. 74, 43-51.

288 Boglione, C.; Gagliardi, F.; Scardi, M.; Cataudella, S., 2001. Skeletal descriptors and 289 quality assessment in larvae and post-larvae of wild-caught and hatchery-reared 290 gilthead sea bream (Sparus aurata L. 1758). Aquaculture 192, 1-22.

292 Cahu, C.; Zambonino Infante, J.; Takeuchi, T., 2003. Nutritional components 293 affecting skeletal development in fish larvae. Aquaculture 227, 245-258.

295 Daoulas, C. H.; Economou, N. A.; Bantavas, I., 1991. Osteological abnormalities in 296 laboratory reared European sea bass (Dicentrachus labrax) fingerlings. Aquaculture $29797,169-180$.

299 Darias, M.J.; Mazurais, D.; Koumoundouros, G.; Glynatsi, N.; Christodoulopoulou, 300 S.; Huelvan, C.; Desbruyeres, E.; Le Gall, M.M.; Quazuguel P.; Cahu, C.L.; 301 Zambonino-Infante, J.L., 2010. Dietary vitamin $D_{3}$ affects digestive system 302 ontogenesis and ossification in European sea bass (Dicentrachus labrax, Linnaeus, 303 1758). Aquaculture 298, 300-307.

305 Dingerkus, G.; Uhler, L. D., 1977. Enzyme clearing of Alcian blue stained whole 306 small vertebrates, for demonstration of cartilage. Stain Technology 52, 229-232. 
308 Faustino, M.; Power, D. M., 1998. Development of osteological structures in the sea

309 bream: vertebral column and caudal fin complex. Journal of Fish Biology 52, 11-22.

311 Faustino, M; Power, D. M., 1999. Development of the pectoral, pelvic, dorsal and

312 anal fins in cultured sea bream. Journal of Fish Biology 54, 1094-1110.

314 Faustino, M.; Power, D. M., 2001. Osteologic development of the viscerocranial

315 skeleton in sea bream: alternative ossification strategies in teleost fish. Journal of

316 Fish Biology 58, 537-572.

318 Fernández, I.; Hontoria, F.; Ortiz-Delgado, J. B.; Kotzamanis, Y.; Estévez, A.;

319 Zambonino-Infante, J. L.; Gisbert, E., 2008. Larval performance and skeletal 320 deformities in farmed gilthead sea bream (Sparus aurata) fed with graded levels of

321 Vitamin A enriched rotifers (Brachionus plicatilis). Aquaculture 283, 102-115.

323 Fernández, I.; Pimentel, M.; Ortiz-Delgado, J. B.; Hontoria, F.; Sarasquete, C.;

324 Estévez, A.; Zambonino-Infante, J. L.; Gisbert, E., 2009. Effect of dietary vitamin A 325 on Senegalese sole (Solea senegalensis) skeletogenesis and larval quality. 326 Aquaculture 295, 250-265.

328 Gavaia, P. J.; Sarasquete, C.; Cancela, M. L., 2000. Detection of mineralized

329 structures in early stages of development of marine Teleostei using a modified

330 alcian blue-alizarin red double staining technique for bone and cartilage. Biotechnic 331 and Histochemistry 75, 79-84.

333 Gavaia, P. J.; Dinis, M. T.; Cancela M. L., 2002. Osteological development and 334 abnormalities of the vertebral column and caudal skeleton in larval and juvenile 
335 stages of hatchery-reared Senegal sole (Solea senegalensis) Aquaculture 211, 305-

336323.

338 Gavaia, P.J.; Simes, D. C.; Ortiz-Delgado, J. B.; Viegas, C. S. B.; Pinto, J. P.; Kelsh,

339 R. N.; Sarasquete, C. M.; Cancela, L. M., 2006. Osteocalcin and matrix Gla protein

340 in zebrafish (Danio rerio) and Senegal sole (Solea senegalensis): Comparative gene

341 and protein expression during larval development through adulthood. Gene

342 Expression Patterns 6, 637-652.

344 Gluckmann, I.; Huriaux, F.; Focant, B.; Vandewalle, P., 1999. Postembryonic

345 development of the cephalic skeleton in Dicentrarchus labrax (Pisces, Perciformes,

346 Serranidae). Bulletin of Marine Science 65, 11-36.

348 Koumoundouros, G.; Gagliardi, F.; Divanach, P.; Boglione, C.; Cataudella, S.;

349 Kentouri, M., 1997a. Normal and abnormal osteological development of caudal fin in

350 Sparus aurata L.

351 fry. Aquaculture 149, 215-226.

353 Koumoundouros, G.; Oran, G.; Divanach, P.; Stefanakis, S; Kentouri, M., 1997b.

354 The opercular complex deformity in intensive gilthead sea bream (Sparus aurata L.)

355 larviculture. Moment of apparition and description. Aquaculture 156, 165-177.

357 Koumoundouros, G.; Divanach, P; Kentouri, M. 2000. Development of the skull in 358 Dentex dentex Osteichthyes: Sparidae). Marine Biology 136, 175-184.

360 Koumoundouros, G.; Maingot, E.; Divanach, P.; Kentouri, M., 2002. Kyphosis in 361 reared European sea bass (Dicentrarchus labrax L.): ontogeny and effects on 362 mortality. Aquaculture 209, 49-58. 
363 Lall, S.P.; Lewis-McCrea, L., 2007. Role of nutrients in skeletal metabolism and

364 pathology in fish, an overview. Aquaculture 267, 3-19

366 Lian, J. B.; Stein, G. S., 1995. Development of the osteoblast phenotype: molecular

367 mechanisms mediating osteoblast growth and differentiation. The lowa Orthopaedic

368 Journal $15,118-40$.

370 Marino, G.; Boglione, C.; Bertolini, B.; Cataudella, S., 1993. Observations on

371 development and anomalies in the appendicular skeleton of sea bass, Dicentrarchus

372 labrax L. 1758, larvae and juveniles. Aquaculture and Fisheries Management 24, $373 \quad 445-456$.

375 Mazurais, D.; Darias, M. J.; Gouillou-Coustans, M. F.; Le Gall, M. M.; Huelvan, C.;

376 Desbruyères, E.; Quazuguel, P.; Cahu, C.; Zambonino-Infante, J. L., 2008. Dietary

377 vitamin mix levels influence the ossification process in European sea bass

378 (Dicentrarchus labrax) larvae. American Journal of Physiology Regulatory

379 Integrative Comparative Physiology 294, R520-7.

381 Mazurais, D.; Glynatsi, G.; Darias, M.J.; Christodoulopoulou, S.; Cahu, C.L.;

382 Zambonino-Infante, J.L.; Koumoundouros, G., 2009. Optimal levels of dietary

383 vitamin A for reduced deformity incidence during development of European sea

384 bass larvae (Dicentrarchus labrax) depend on malformation type. Aquaculture 294, $385 \quad 262-270$.

387 Nishimoto, S.K.; Araki, N.; Robinson, D.F.; Waite, H.J., 1992. Discover of Bone

388 Carboxyglutamic Acid Protein in Mineralized Scales. The Journal of Biological 389 Chemistry 267, 11600-11605. 
391 Nishimoto, S.K.; Waite, H.J.; Nishimoto, M.; Kriwacki, R.W., 2003. Structure,

392 Activity, and Distribution of Fish Osteocalcin. The Journal of Biological Chemistry

$393 \quad 278(14), 11843-11848$.

394

395 Park, E. H.; Kim, D. S., 1984. A procedure for staining cartilage and bone of whole

396 vertebrate larvae while rendering all other tissues transparent. Stain Technology 59,

$397 \quad 269-72$.

398

399 Potthoff, T., 1984. Clearing and staining techniques. In: Ontogeny and sytematics of 400 fishes. H.G. Moser, W.J. Richards, D.M. Cohen, M.P. Fahay, A.W. Kendall, Jr., and

401 S.L. Richardson (Eds). The American Society of Ichthyologists and Herpetologists.

402 Spec. Publ. No. 1. pp 35-37.

403

404 Sfakianakis, D.G.; Koumoundouros, G.; Divanach, P.; Kentouri, M., 2004.

405 Osteological development of the vertebral column and of the fins in Pagellus

406 erythrinus (L. 1758). Temperature effect on the developmental plasticity and

407 morpho-anatomical abnormalities. Aquaculture 232, 407-424.

408

409 Swaminathan, R., 2001. Biochemical markers of bone turnover. Clinica Chimica 410 Acta 313, 95-105.

412 Taylor, W. R.; Van Dyke, G. C., 1985. Revised procedures for staining and clearing 413 small fishes and other vertebrates for bone and cartilage study. Cybium 9, 107-119.

415 Wagemans, F.; Focant, B.; Vandewalee, P., 1998. Early development of the 416 cephalic skeleton in the turbot. Journal of Fish Biology 52, 166-204. 
420 Figure 1. Alcian blue-alizarin red double stained European sea bass larvae. A) 4.5

$421 \mathrm{~mm} \mathrm{TL}$, only cartilaginous structures were observed; B) $6.4 \mathrm{~mm} \mathrm{TL}$, the first signs of 422 ossification appeared; C and D) $8.3 \mathrm{~mm} \mathrm{TL}$, the vertebral colum started to ossify 423 (magnified picture shows that mineralization proceeded ventrad); E) $12.8 \mathrm{~mm} \mathrm{TL}$, 424 the vertebrae centra are more mineralized; F) $15.5 \mathrm{~mm} \mathrm{TL}$, ossification is much 425 more advanced, including the cephalic region, vertebral column, caudal fin complex 426 and two thirds of pectoral, dorsal, ventral and caudal fins. As observed, this double 427 staining procedure allows to describe the skeletal development of the European sea 428 bass. $\mathrm{Cl}$, Cleithrum; De, dentary, HS, Hyosymplectic; Mc, Meckel's cartilage; Mx, 429 maxilay; $\mathrm{Q}$, quadrate; Sc, sclerotic. A-C, scale bars are equal to $0.5 \mathrm{~mm}$. D-F, scale 430 bars are equal to $1 \mathrm{~mm}$.

432 Figure 2. Alcian blue-alizarin red double stained European sea bass larvae showing 433 several malformations (indicated by arrows). A) Pugheadness in the skull and 434 formation of cartilaginous tissue in the vertebrae; B) Elongation of the lower jaw; C) 435 Fusion of epurals and deformation of the uroneural; D) The same malformations of 436 cartilaginous structures are also found after their mineralization; E) Kyphosis of the 437 vertebral column. Scale bars are equal to $1 \mathrm{~mm}$.

439 Figure 3. Level of ossification (red pixels/larvae) and relative osteocalcin gene 440 expression during the European sea bass larval development. The mineralization 441 degree in bony tissue increased from $6.4 \mathrm{~mm}$ TL onwards, coinciding with the 442 detection of the first ossified structures (dentary, maxillas and cleithrum, see Fig. 1). 443 Mineralization remained limited to the skull until $8.3 \mathrm{~mm}$ TL. From 8.3 to $15.5 \mathrm{~mm}$ $444 \mathrm{TL}$, the mineralized bone gradually progressed throughout the vertebral column (see 445 Fig. 1). Osteocalcin expression and ossification process followed similar tendencies. 
446 The values in lines represent means and bars are standard deviation. Four 447 replicates of $40-50$ samples per replicate and sample point.

448

449 
450 Table 1. Incubation times of the double-staining protocol used in each larval group

451 according to the European sea bass larval development

\begin{tabular}{lccc}
\hline Larval groups & a & b & c \\
\hline Larval age & $7-15 \mathrm{dph}$ & $17-25 \mathrm{dph}$ & $30-40 \mathrm{dph}$ \\
Total length & $4.5-6.4 \mathrm{~mm}$ & $6.7-8.3 \mathrm{~mm}$ & $12.8-15.5 \mathrm{~mm}$ \\
\hline Incubation times for each & & & \\
protocol stage & & & \\
\hline Cartilage staining & $30 \mathrm{~min}$. & $60 \mathrm{~min}$. & $24 \mathrm{~h}$ \\
Bleaching & $25 \mathrm{~min}$. & $30 \mathrm{~min}$. & $60 \mathrm{~min}$. \\
Clearing & - & - & $20 \mathrm{~h}$ \\
Bone staining & $30 \mathrm{~min}$. & $20 \mathrm{~h}$ & $20 \mathrm{~h}$ \\
Washing & $5 \mathrm{~min}$. & $5 \mathrm{~min}$ & $2 \times 5 \mathrm{~min}$. \\
\hline
\end{tabular}

452

453 Darias et al., JAI-Bo-21, Table 1

454 
A

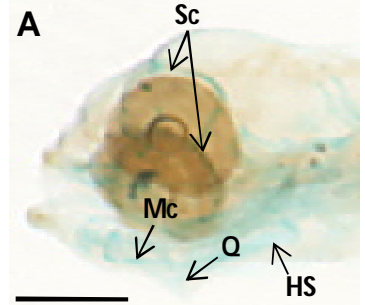

B

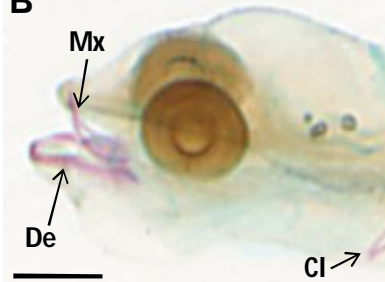

C

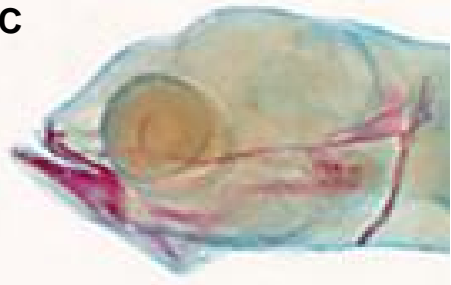

$\mathrm{Cl} \lambda$

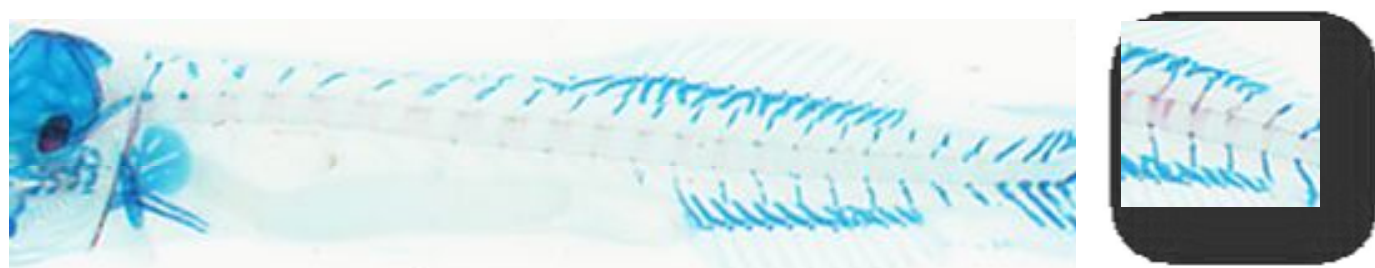

D
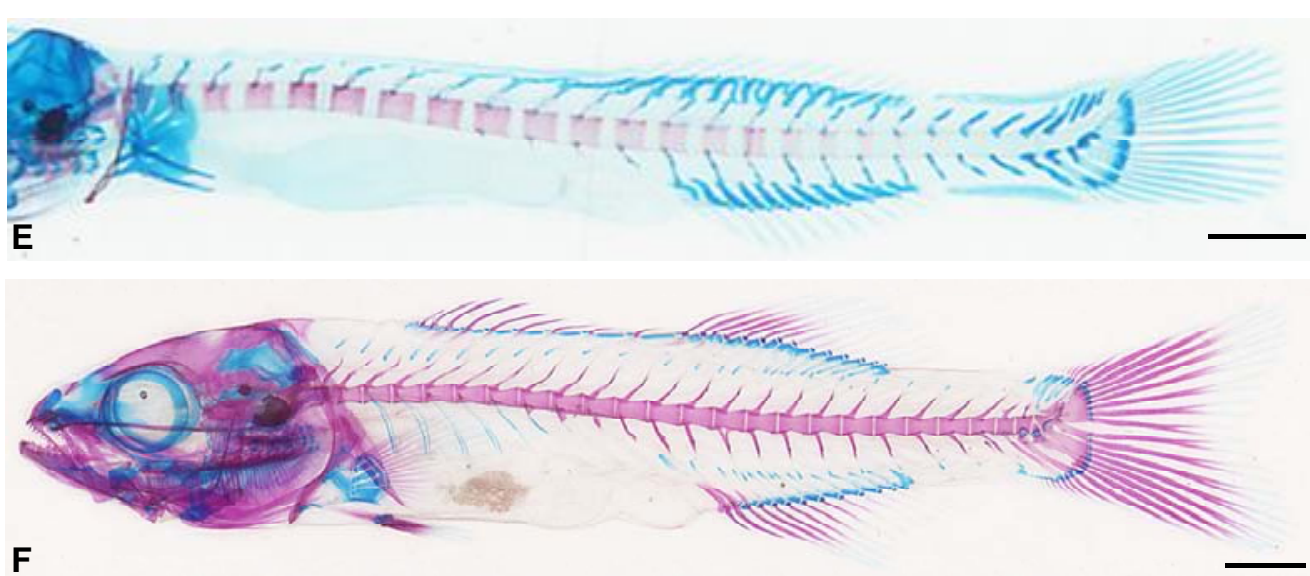


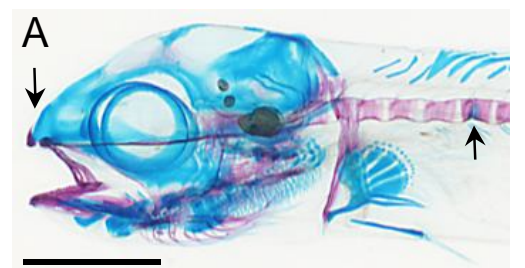

C

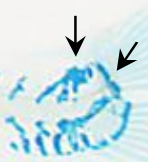

Darias et al., JAI-Bo-21, Figure 2
B

D

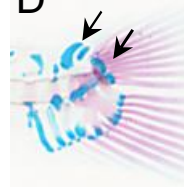

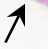
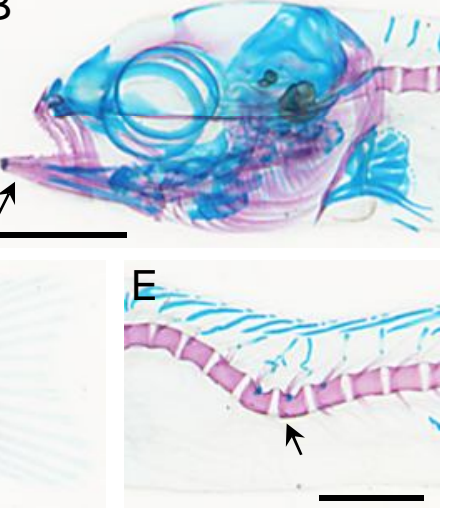

458 


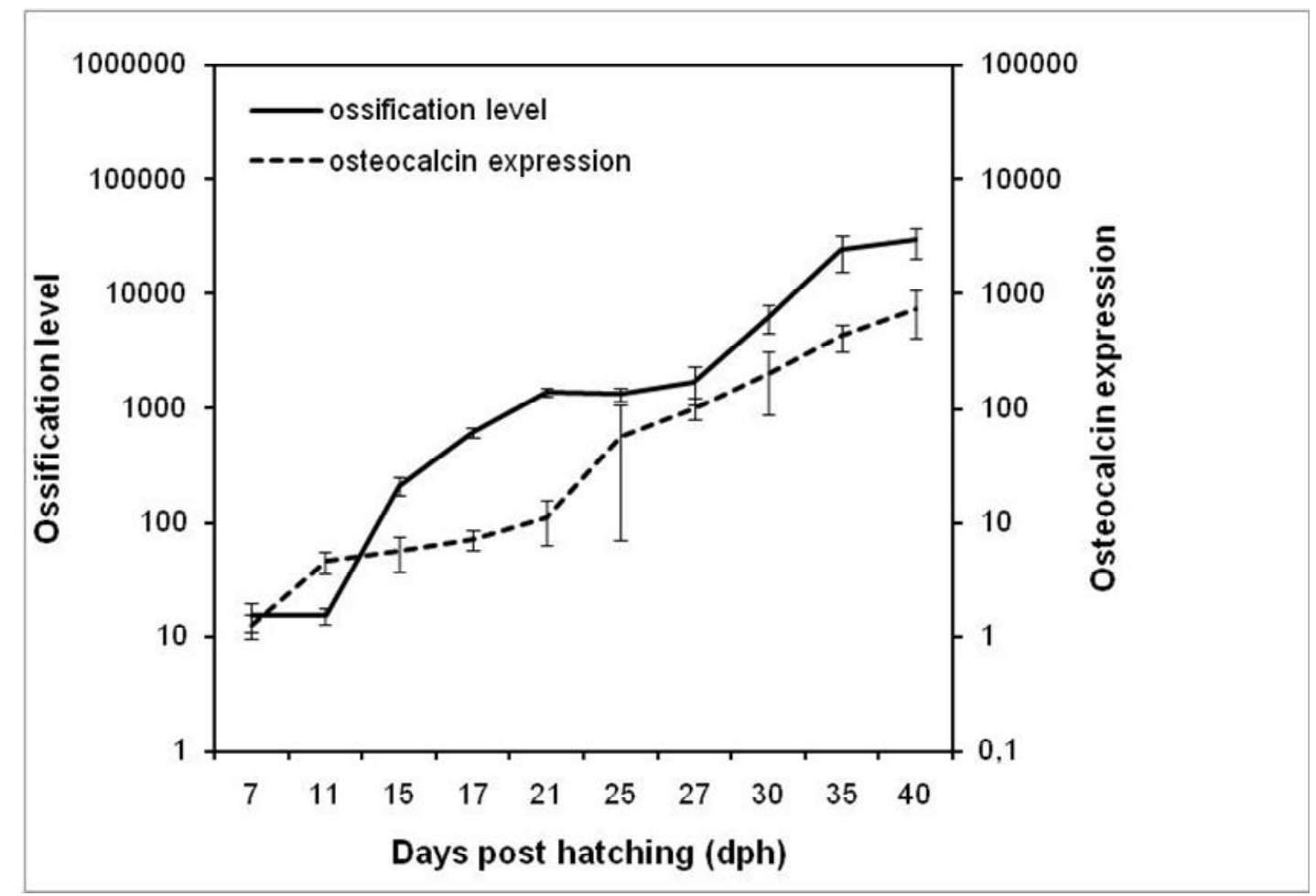

